\title{
Expansions in terms of Parabolic Cylinder Functions
}

\author{
By T. M. Cherry
}

( Received 3rd October, 1946. Read 1st November, 1946.)

1. When the plane wave equation is expressed in terms of parabolic co-ordinates $x, y$, the variables are separable, and the elementary solutions have the form

$$
D_{-t+i \mu}\left(x e^{ \pm i \pi / 4}\right) \quad D_{-i-i \mu}(y e \pm i \pi / 4),
$$

where $x, y, \mu$ are real. In this context, therefore, the functions $D_{\nu}(z)$ which are directly significant are those where amp $z= \pm \pi / 4$ and $\nu+\frac{1}{2}$ is purely imaginary, rather than those where $z$ is real and $\nu$ is a positive integer. The expansion of an arbitrary function in terms of the latter sort of $D$-function (substantially, in terms of Hermite polynomials) is well known. This paper is concerned with the expansion in terms of the former sort of $D$-function. The result obtained is: if $f(x)$ is of bounded variation in any finite interval of the real variable $x$ and is absolutely integrable in $(-\infty, \infty)$, then

$$
\begin{aligned}
&-4 \pi i f(x)=\int_{-\frac{1}{2}-i \infty}^{-\frac{1}{2}+i \infty} \frac{e^{\frac{t}{(}\left(\nu+\frac{1}{2}\right) \pi i}}{\sin \nu \pi} d \nu \int_{-\infty}^{\infty} f(t) \\
&\left\{D_{\nu}(x \eta) D_{-\nu-1}(\bar{\eta})+D_{\nu}(-x \eta) D_{-\nu-1}(-t \bar{\eta})\right\} d t,
\end{aligned}
$$

where $\eta=e^{\pi i / 4}, \bar{\eta}=e^{-\pi i / 4}$.

To put this result in "real" form, let $\nu=-\frac{1}{2}+i \mu$, and let

$$
\begin{aligned}
& \phi_{\mu}(x)=\pi^{-\frac{1}{2}} 2^{-\frac{1}{2} \nu-1} \Gamma\left(\frac{1}{2}-\frac{1}{2} \nu\right)\left\{D_{\nu}(x \eta)+D_{\nu}(-x \eta)\right\}, \\
& \psi_{\mu}(x)=-\pi^{-\frac{1}{2}} 2^{-\frac{1}{2} \nu-\frac{3}{2}} e^{-\pi i / 4} \Gamma\left(-\frac{1}{2} \nu\right)\left\{D_{\nu}(x \eta)-D_{\nu}(-x \eta)\right\} .
\end{aligned}
$$

Then $\phi_{\mu}(x),=1+\ldots$ and $\psi_{\mu}(x),=x+\ldots$ are respectively even and odd, and are real when $x, \mu$ are real; and

$$
\begin{aligned}
& 2 \sqrt{ } 2 f(x)=\int_{-\infty}^{\infty} \frac{e^{-\frac{1}{2} \mu \pi} d \mu}{\cosh \mu \pi} \int_{-\infty}^{\infty} f(t)\left\{\frac{\phi_{\mu}(x) \phi_{-\mu}(i t)}{\Gamma\left(\frac{3}{4}+\frac{1}{2} i \mu\right) \Gamma\left(\frac{3}{4}-\frac{1}{2} i \mu\right.}\right) \\
& \left.-\frac{2 i \psi_{\mu}(x) \psi_{-\mu}(i t)}{\Gamma\left(\frac{1}{4}+\frac{1}{2} i \mu\right) \Gamma\left(\frac{1}{4}-\frac{1}{2} i \mu\right)}\right\} d t .
\end{aligned}
$$

Using a different notation, W. Magnus ${ }^{1}$ has proved formulæ which are substantially equivalent to (A), for the case where $f(x)$ is an analytic function which has for $x \sim \pm \infty$ an asymptotic expansion of the form

I W. Magnus, Jahresbericht Deutsch. Math. Verein., 50 (1940), 140-161. The formula (A) was proved by A. Erdélyi about 1934, but was not published. 


$$
f(x) \sim e^{-i x^{2 / 4}}\left(\frac{c_{1}}{x}+\frac{c_{2}}{x^{2}}+\frac{c_{3}}{x^{3}}+\ldots\right)
$$

The formula (A) can be put in a variety of other forms, some of which are given in equations $(15)-(18)$ below. All of these are asymmetrical as regards $\nu \sim i \infty, \nu \sim-i \infty$; the essential reason for the asymmetry is that, for $|x|<2|\mu|^{\frac{1}{2}}$, the functions $D_{-1+i \mu}( \pm x \eta)$ are oscillating (roughly like $\exp \left( \pm i x \sqrt{ } \mu^{\prime}\right)$ ) for $\mu=-\mu^{\prime}<0$, but nonoscillating (roughly like $\exp ( \pm x \sqrt{ } \mu)$ ) for $\mu>0$.

The inner integrals in (A) are absolutely convergent if $x^{-\frac{1}{2}} f(x)$ is absolutely integrable, and it is possible that (A) is valid under this condition; but I have not proved this. I do, however, prove (A) when, for $x \sim \infty$,

$$
f(x) \sim e^{-i x 2 / 4}\left(\frac{c_{1}}{x^{a}}+\frac{c_{2}}{x^{1 \cdot \cdot a}}\right),
$$

with $a>\frac{1}{2}$; and for $x \sim-\infty, f(x)$ has a similar form; this condition is somewhat wider than Magnus's condition (C).

In the concluding section there are some examples of representation of continuous and discontinuous functions in the form (A).

The following properties ${ }^{1}$ of $D_{v}(z)$ are required:

(i) For $\nu \sim \infty$,

$$
D_{\nu}(z)=2^{-\frac{1}{2}} \exp \left\{\frac{1}{2} \nu \log (-\nu)-\frac{1}{2} \nu-(-v)^{\frac{1}{2}} z\right\} \cdot\left\{1+O\left(\nu^{-\frac{1}{2}}\right)\right\}
$$

uniformly when $z$ is bounded and $|\operatorname{amp}(-v)| \leqq \frac{1}{2} \pi$; here, and always in the sequel, $\log (-v)$ and $(-v)^{\frac{1}{2}}$ denote principal values.

(ii) Let

$$
z=2(-\nu)^{\frac{1}{2}} \sin \alpha
$$$$
\alpha=\beta+i \gamma \text {. }
$$

For the values of $z, \nu$ with which we shall be concerned, we take the principal determination of $\alpha$, for which $|\gamma| \leqq \frac{1}{2} \pi$; this specification is without ambiguity provided $|\gamma|<\frac{1}{2} \pi$ while when $|\gamma|=\frac{1}{2} \pi$ the proper specification follows by continuity, as will be seen in $\S 3$.

With this determination of a, for $\mathrm{Rl} \nu \leqq 0,|\nu| \geqq N>0$ and $\operatorname{Rl} z \geqq|\operatorname{Im} z|$,

${ }^{1}$ It is likely that the results (i), (ii) are not new, but I know of no reference; they may be proved from the formula

$$
D_{\nu}(z)=(2 \pi)^{-\frac{1}{2} e^{-\frac{1}{2}} \nu \pi i} \int_{i c-\infty}^{i c+\infty} \exp \left(\frac{1}{4} z^{2}-\frac{1}{2} t^{2}+i z t^{v}\right) t \nu d t \quad(c>0)
$$

by the saddle-point method. In Proc. London Math. Soc. (2), 17 (1918), 116, Watson proves results like (3), but his work does not cover the case where amp $v$ is near $\pm \frac{1}{2} \pi$. For (iii) see Whittaker and Watson, Modern Anclysis (4th ed.) \$16.52. 
$\left|D_{\nu}(z)\right| \leqq A_{N}\left|\exp \left\{\frac{t}{2} \nu \log (-\nu)-\frac{1}{2} \nu+\nu(\sinh a \cosh a+a)\right\}\right| \times$ $\times \begin{cases}|\nu|^{\frac{1}{b}}, & \text { for }\left|1+e^{-2 a}\right| \leqq|\nu|^{-\frac{1}{1}} \\ \left|2 e^{\alpha} \operatorname{sech} a\right|^{\frac{1}{2}}, & \text { for }\left|1+e^{-2 a}\right|>|\nu|^{-\frac{1}{3}},\end{cases}$

where the constant $A_{N}$ depends only on $N$.

(iii) For any $z, \nu$

$$
\begin{aligned}
& D_{\nu}(z)=e^{\nu \pi i} D_{\nu}(-z)+(2 \pi)^{\frac{1}{2}} e^{\frac{z}{3}(\nu+1) \pi i} D_{-\nu-1}(-i z) / \Gamma(-\nu), \\
& D_{\nu}(z)=e^{-\nu \pi i} D_{\nu}(-z)+(2 \pi)^{\frac{1}{t}} e^{-\frac{1}{2}(\nu+1 ; \pi} D_{-\nu-1}(i z) / \Gamma(-\nu) .
\end{aligned}
$$

2. Let $a<x<b$ be real constants; $f(t)$ a function of a real variable $t$, of bounded variation in $a \leqq t \leqq b$; and $\theta$ any constant whose amplitude is between $\pm \frac{1}{4} \pi$ (inclusive). Consider the function

$$
F(\nu, x)=\frac{\Gamma(-v)}{(2 \pi)^{\frac{1}{2}}}\left\{D_{\nu}(x \theta) \int_{a}^{x} f(t) D_{\nu}(-t \theta) d t+D_{\nu}(-x \theta) \int_{x}^{b} f(t) D_{v}(t \theta) d t\right\} .
$$

This is an analytic function of $\nu$, regular except at the poles of $\Gamma(-\nu)$, and we are to integrate it along a path equivalent to a large semicircle in the half-plane $\mathrm{Rl} \nu \leqq 0$.

From (1), together with Stirling's theorem, we have for $\nu \sim \infty$ with $\mathrm{Rl} \nu \leqq 0$,

$(2 \pi)^{-t} \Gamma(-v) D_{\nu}(t \theta) D_{\nu}(-x \theta)=\frac{1}{2}(-v)^{-\frac{t}{2}} \exp \left\{-(-v)^{-\frac{1}{3}} \theta(t-x)\right\} \cdot\left\{1+O\left(\nu^{-\frac{1}{t}}\right)\right\}$.

Hence, since $f(t)$ is of bounded variation,

$$
\begin{aligned}
& (2 \pi)^{-\frac{1}{t}} \Gamma(-\nu) D_{\nu}(-x \theta) \int_{x}^{b} f(t) D_{\nu}(t \theta) d t \\
& =\frac{1}{2 \nu \theta}\left[f(b-0) e^{-x(b-x)}-f(x+0)\right] \\
& \quad-\frac{1}{2 \nu \theta} \int_{x}^{b} e^{-n(t-x)} d f(t)+\frac{1}{2(-\nu)^{\frac{1}{3}}} \int_{x}^{b} e^{-n(t-x)} f(t) O\left(\nu^{-t}\right) d t,
\end{aligned}
$$

where we have put $(-\nu)^{\frac{1}{}} \theta=n$.

Since both $(-v)^{\frac{1}{2}}$ and $\theta$ have amplitudes between $\pm \frac{1}{4} \pi$, and $x<t<b$, the real parts of $n$ and of $n(t-x)$ are non-negative. Writing then $V(c, d)$ for the total variation of $f(t)$ in the interval $(c, d)$, $C /|\nu|^{\frac{1}{2}}$ for the upper bound of $\left|f(t) O\left(\nu^{-\frac{1}{2}}\right)\right|$ in $(x, b)$ and $n_{1}=\operatorname{Rl} n$ $=\operatorname{Rl}\left[(-\nu)^{\prime} \theta\right]$, we have

$$
\begin{aligned}
& \left|\frac{1}{2 \nu \theta} \int_{x}^{b} \exp \left\{-(-\nu)^{\frac{1}{2}} \theta(t-x)\right\} d f(t)\right| \leqq \frac{V(x, x+\delta)}{|2 \nu \theta|}+\frac{V(x, b)}{|2 \nu \theta|} e^{-\delta n_{1},} \\
& \left|\frac{1}{2(-\nu)^{\frac{1}{2}}} \int_{x}^{b} \exp \left\{-(-\nu)^{\frac{1}{2} \theta} \theta(t-x)\right\} f(t) O\left(\nu^{-\frac{1}{2}}\right) d t\right| \leqq \frac{C}{2|\nu|}\left\{\delta+(b-x) e^{-\delta n_{1}}\right\}
\end{aligned}
$$

where $\delta$ is any small positive number. The contribution of these two 
terms to the integral of $F$ round the semi-circle $|\nu|=r$ in the halfplane $\mathrm{Rl} \nu \leqq 0$ is hence less than

$$
\frac{1}{2} \pi\{V(x, x+\delta) /|\theta|+C \delta\}+\phi(r, \delta),
$$

where, by Jordan's lemma, $\phi(r, \delta) \rightarrow 0$ as $r \rightarrow \infty$, for any fixed $\delta$. By first choosing $\delta$ sufficiently small and then $r$ sufficiently large, we see that these two terms give a zero integral in the limit as $r \rightarrow \infty$; and by Jordan's lemma the same applies to the first term on the right of (8). Hence (8) gives

$\lim _{r \rightarrow \infty} \int_{-c-i r}^{-c+i r}(2 \pi)^{-i} \Gamma(-\nu) D_{\nu}(-x \theta) d \nu \int_{x}^{b} f(t) D_{\nu}(t \theta) d t=\frac{\pi i}{2 \theta} f(x+0)$,

where $c$ is any positive constant.

For later reference we note that, if the integral on the left of (8) had had a lower limit $a$ exceeding $x$ (instead of the lower limit $x$ ), every term on the right would have carried an exponential factor. Hence:

$$
\begin{gathered}
\text { if } x<a<b, \text { then for }|\nu| \text { sufficiently large, with } \mathrm{Rl} \mathrm{l}_{\nu} \leqq 0 \\
(2 \pi)^{-\frac{1}{2}} \Gamma(-\nu) D_{\nu}(-x \theta) \int_{a}^{b} f(t) D_{\nu}(t \theta) d t \mid \leqq C^{1} \nu^{-1} \exp \left\{-(-\nu)^{\frac{1}{2}} \theta(a-x)\right\},
\end{gathered}
$$

where $C$ is a constant depending on $x, a, b$ but not on $\nu$.

The first term on the right of (6) gives a result similar to (9); hence if $f(t)$ is of bounded variation in $a \leqq t \leqq b$, and $a<x<b$, and $\theta$ is a constant whose amplitude is between $\pm \frac{1}{4} \pi$ (inclusive), then

$$
\frac{\pi i}{2 \theta}\{f(x-0)+f(x+0)\}=\lim _{r \rightarrow \infty} \int_{-\frac{1}{2}-i r}^{-\frac{1}{2}+i r} F(\nu, x) d \nu
$$

where $F(\nu, x)$ is defined by $(6)$.

If we abbreviate by writing $f(x)$ for $\frac{1}{2}\{f(x-0)+f(x+0)\}$, this formula is equivalent to

$$
\begin{aligned}
& \pi i f(x)=\lim _{r \rightarrow \infty} \int_{-\frac{1}{2}-i r}^{-\frac{1}{2}+i r}\left\{(2 \pi)^{-\frac{1}{2}} \Gamma(-\nu) \theta D_{v}(-x \theta) \int_{a}^{b} f(t) D_{v}(t \theta) d t\right. \\
&\left.+\int_{a}^{x} \phi(\nu, \theta, x, t) d t\right\} d \nu,
\end{aligned}
$$

where $\phi(\nu, \theta, x, t)=(2 \pi)^{-1} \Gamma(-\nu) \theta\left\{D_{\nu}(x \theta) D_{\nu}(-t \theta)-D_{\nu}(-x \theta) D_{\nu}(t \theta)\right\}$. (12) Since the integrand is regular in $\mathrm{Rl}_{\nu}<0$, the integral can be taken along the line $\mathrm{Rl} \nu=-\frac{1}{2}$.

We have now to combine (11) with a similar formula so as to eliminate the term in $\int_{a}^{x} \phi d t$. For this purpose, write

$$
\eta=e^{\frac{1}{2 \pi i}}, \quad \bar{\eta}=e^{-\frac{1}{4} \pi i},
$$

take $\theta=\eta$ in (11), and for the companion take $\theta=\bar{\eta}$ in (11) and change 
the variable by putting $\nu=-\nu^{\prime}-1$. The companion is thus

$$
\begin{array}{r}
\pi i f(x)=\lim _{r \rightarrow \infty} \int_{-\underline{-i r}}^{-\frac{1}{2}+i r}\left\{(2 \pi)^{-\frac{1}{2}} \Gamma(\nu+1) D_{-\nu-1}(-x \bar{\eta}) \int_{a}^{b} f(t) D_{-\nu-1}(t \eta) d t\right. \\
\left.+\int_{a}^{x} \phi(-\nu-1, \bar{\eta}, x, t) d t\right\} d \nu .
\end{array}
$$

Now from (12) we have, on substituting from (5) for $D_{v}(-t \eta)$, $D_{\nu}(-x \eta)$,

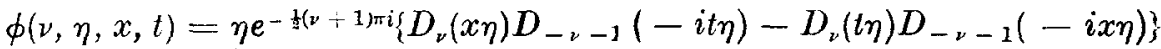

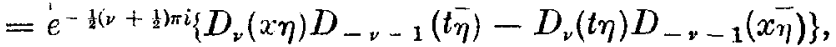

since $\eta=e^{\frac{1}{\pi \pi i}}=i \vec{\eta}$. With a similar substitution from (4), (12) gives $\phi(v, \bar{\eta}, x, t)=\bar{\eta} e^{\left.\frac{1}{(2 v}+1\right) \pi i}\left\{D_{v}(x \bar{\eta}) D_{-v-1}(i t \bar{\eta})-D_{v}(t \bar{\eta}) D_{-v-1}(i x \eta)\right\}$

$$
=e^{\frac{3}{2}\left(\nu+\frac{1}{2}\right) \pi i}\left\{D_{\nu}\left(x_{\eta}\right) D_{-\nu-1}(i \eta)-D_{v}(i \eta) D_{-v-1}(x \eta)\right\} ;
$$

and hence $\quad \phi(\nu, \eta, x, t)=-\phi(-v-1, \eta, x, t)$.

Hence, putting $\theta=\eta$ in (11) and adding to (14), we have

THEOREM 1. If $f(t)$ is of bounded variation in $a \leqq t \leqq b$, and $\eta=e^{1 \pi i}$, $\eta=e^{-i}\{\pi i$, then, for $a<x<b$,

$$
\begin{aligned}
(2 \pi): i f(x)=\int_{-\frac{1}{-1}-i \infty}^{-i+i} d \nu \int_{a}^{b} f(t)\left\{\Gamma(-\nu) \eta D_{\nu}(-x \eta) D_{\nu}(\ell \eta)\right. \\
\left.+\Gamma(\nu+1) \bar{\eta} D_{-\nu-1}(-x \eta) D_{-\nu-1}(t \eta)\right\} d t,
\end{aligned}
$$

where the $\boldsymbol{\nu}$-integral is a Cauchy principal value.

The formula (15) is capable of various transformations by means of (4), (5). For instance, we can express $D_{\nu}(t \eta)$ in terms of $D_{-v-1}\left(\overline{t_{\eta}}\right)$, $D_{-v-1}(-t \bar{\eta})$, and $D_{-\nu-1}(-x \bar{\eta})$ in terms of $D_{v}(x \eta), D_{\nu}(-x \eta)$. This gives a common factor $\Gamma(-v) \Gamma(\nu+1)$, the terms in $D_{\nu}(-x \eta) D_{-\nu=1}(t \eta)$ cancel, and we obtain

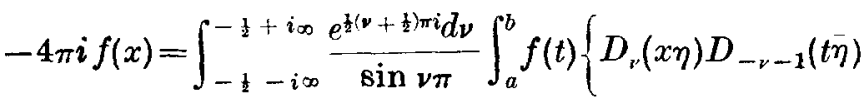

$$
\begin{aligned}
& \left.+D_{v}\left(-x_{\eta}\right) D_{-,-1}(-t \eta)\right\} d t .
\end{aligned}
$$

If here we substitute for $D_{-v-1}\left(t_{\eta}\right)$ from (4) and for $D_{v}(-x \eta)$ from (5), we obtain

$$
\begin{aligned}
(2 \pi)^{\S} i f(x)=\int_{-\frac{1}{2}-i \infty}^{-\frac{1}{2}+i \infty} d \nu \int_{a}^{b} f(t)\left\{\Gamma(-\nu) \eta D_{\nu}(x \eta) D_{\nu}(-t \eta)\right. \\
\left.+\Gamma(\nu+1) \bar{\eta} D_{-\nu-1}(x \bar{\eta}) D_{-\nu-1}(-\bar{\eta})\right\} d t,
\end{aligned}
$$

which is similar to (15) but with the signs changed in the arguments 
of the $D$-functions. If here we substitute for $D_{v}(-t \eta), D_{-v-1}(x \vec{\eta})$, we obtain

$$
\begin{aligned}
&(2 \pi)^{\frac{3}{3}} i f(x)=\int_{-\frac{1}{2}-i \infty}^{-\frac{1}{2}+i \infty} e^{\nu \pi i} d \nu \int_{a}^{b} f(t)\left\{\Gamma(-\nu) \eta D_{\nu}(x \eta) D_{\nu}(t \eta)\right. \\
&-\Gamma(\nu+1) \bar{\eta} D_{-\nu-1}(-x \widetilde{\eta}) D_{-v-1}\left(-t_{\eta}\right\} d t,
\end{aligned}
$$

and there is an analogue of this with the signs of the arguments of the $D$-functions changed. It is to be emphasised that the integrands of (15)-(18) are identical, and that there are no forms with identical integrands which are analogous to $(16)$ and (18) but with the initial factors $e^{-\frac{1}{2}(\nu+1) \pi i}, e^{-\nu \pi i}$. In fact, as will be seen, the integrals (15)-(18) are more rapidly convergent for $\nu \sim i \infty$ than for $\nu \sim-i \infty$ (so that, incidentally, they exist in the ordinary sense and not merely as principal values), and the formulæ in which the behaviours at $\nu \sim \pm i \infty$ are interchanged are derived from (15)-(18) by the change of variable $\nu=-\nu^{\prime}-1$.

3. With a view to extending Theorem 1 to an infinite $x$-range we now develop certain consequences of the inequality (3). We write

$$
\begin{aligned}
& (-\nu)^{\frac{1}{2}}=\mu^{\frac{1}{3}} e^{i \theta}, \quad M=\frac{1}{2} \nu \log (-\nu)-\nu, \\
& E=E(\nu, x)=\operatorname{Rl}\{\nu(\sinh \alpha \cosh \alpha+a)\},
\end{aligned}
$$

where $\mu^{\frac{1}{2}}$ is real and positive and $-\frac{1}{4} \pi \leqq \theta \leqq \frac{1}{4} \pi$, and we take

$$
z=x e^{\pi i / 4},
$$

where $x$ is real. Then from (2), since $\alpha=\beta+i \gamma$ and $x$ is real, we find $e^{\beta} \sin \left(\frac{1}{4} \pi-\theta-\gamma\right)=e^{-\beta} \sin \left(\frac{1}{4} \pi-\theta+\gamma\right)=\sqrt{ } \frac{1}{2}(\cos 2 \gamma-\sin 2 \theta),(21)$

and from (20), (21)

$$
\begin{aligned}
\frac{x}{\mu^{\frac{1}{2}}}=\frac{\sin 2 \gamma}{\sqrt{\frac{1}{2}(\cos 2 \gamma-\sin 2 \theta)}} & =\frac{e^{\beta} \sin 2 \gamma}{\sin \left(\frac{1}{4} \pi-\theta+\gamma\right)} \\
& =\frac{e^{-\beta} \sin 2 \gamma}{\sin \left(\frac{1}{4} \pi-\theta-\gamma\right)},
\end{aligned}
$$

$-E / \mu=\frac{1}{4} e^{2 \beta} \cos 2(\theta+\gamma)-\frac{1}{4} e^{-2 \beta} \cos 2(\theta-\gamma)+\beta \cos 2 \theta-\gamma \sin 2 \theta$

$$
=\frac{1}{2} \sin 2 \gamma+\beta \cos 2 \theta-\gamma \sin 2 \theta \text {. }
$$

Hence $\frac{d \beta}{d \gamma}=\frac{\cos 2 \theta}{\cos 2 \gamma-\sin 2 \theta}, \quad \frac{1}{(2 \mu)^{\frac{1}{2}}} \frac{d x}{d \gamma}=\frac{1-2 \cos 2 \gamma \sin 2 \theta+\cos ^{2} 2 \gamma}{(\cos 2 \gamma-\sin 2 \theta)^{\frac{3}{2}}}$.

It follows that, for $-\frac{1}{4} \pi<\theta<\frac{1}{4} \pi$, i.e. for $\mathrm{Rl} \nu<0$, both $\beta$ and $x$ run monotonically from $-\infty$ to $x$ as $\gamma$ runs from $-\left(\frac{1}{4} \pi-\theta\right)$ to $\left(\frac{1}{4} \pi-\theta\right)$, and as (23) gives

$$
-\mu^{-1} d E / d \gamma=\cos 2 \gamma-\sin 2 \theta+\cos 2 \theta d \beta / d \gamma
$$

$E$ then decreases steadily from $\infty$ to $-\infty$. Also $\gamma=0$ gives $\beta=x=E=0$. 
Hence, if $\mathrm{Rl} v<0$ and $x_{1}<x_{2}<0<x_{3}<x_{4}$, then

$$
E\left(x_{1}\right)>E\left(x_{2}\right)>0>E\left(x_{3}\right)>E\left(x_{4}\right)
$$

where $E(x)$ specifies the magnitude of $D_{\nu}\left(x e^{\pi i / 4}\right)$ according to (3), (20).

In the limiting case where amp $\nu=-\frac{1}{2} \pi, \theta=\pi / 4, \beta$ runs from $-\infty$ to $\infty$ with $x$ while $\gamma, E$ both remain zero. In the limiting oase where $\operatorname{amp} v=\pi / 2, \quad \theta=-\pi / 4$, we have:

$$
\begin{aligned}
& \text { for } x<-2 \mu^{\frac{1}{2}}: \quad \gamma=-\frac{\pi}{2}, \quad-\infty<\beta<0, \quad \text { and } E=\frac{1}{2} \mu \text {; } \\
& \text { for }-2 \mu^{\frac{1}{2}}<x<2 \mu^{\frac{5}{2}}: \quad \beta=0, \quad-\frac{\pi}{2}<\gamma<\frac{\pi}{2}, \quad \text { and } \frac{\mu}{2}>E>-\frac{\mu}{2} \text {; } \\
& \text { for } x>2 \mu^{\frac{1}{}}: \quad \gamma=i \frac{\pi}{2}, \quad 0<\beta<\infty, \quad \text { and } E=-\frac{\mu}{2},
\end{aligned}
$$

the three forms of (22) serving for the respective ranges.

Regarding the second factor in (3), the statement is that we can take the smaller of $\left|2 e^{a} \operatorname{sech} a\right|^{1},|\nu|^{1}$, and the latter choice is necessary only when $\left|1+e^{-2 a}\right| \leqq|\nu|-\frac{1}{3}$ i.e. when either $a-\frac{1}{2} \pi i$ or $a+\frac{1}{2} \pi i$ is $O\left(v^{-3}\right)$. Since $\alpha=\beta+i \gamma$ and the extreme values of $\gamma$ are $\pm\left(\frac{1}{4} \pi-\theta\right)$, it follows that the choice $\left|2 e^{a} \operatorname{sech} \alpha\right|^{\frac{1}{*}}$ is permissible when $0 \leqq \theta \leqq \frac{1}{4} \pi$, i.e. when both Rlv and $\operatorname{Im} \nu$ are non-positive. From (21) we then have

$$
\left|2 e^{\alpha} \operatorname{sech} \alpha\right|^{2}=\frac{\sin ^{2}\left(\frac{1}{4} \pi-\theta+\gamma\right)}{\cos ^{2} 2 \theta+(\cos 2 \gamma-\sin 2 \theta)^{2}},
$$

and hence, for $0 \leqq \frac{1}{4} \pi-\theta \leqq \frac{1}{4} \pi$ and $|\gamma| \leqq \frac{1}{4} \pi-\theta$,

Hence (3) gives:

$$
\left|2 e^{a} \operatorname{sech} a\right|^{2} \leqq \frac{\sin ^{2}\left(\frac{1}{2} \pi-2 \theta\right)}{\cos ^{2} 2 \theta}=1
$$

If $\mathrm{Rl} \nu \leqq 0,|\nu| \geqq N>0$ and $x$ is real, then

$$
\left|D_{v}(x \eta)\right| \leqq A_{N}\left|v^{3} \exp (M+E(v, x))\right|,
$$

where $M, E(\nu, x)$ are defined by (19), (20). If also $x \geqq 0$, then

$$
\left|D_{v}(x \eta)\right| \leqq A_{N}\left|\nu^{1} \exp M\right| \text {. }
$$

If $\mathrm{Rl} \nu \leqq 0, \operatorname{Im} \nu \leqq 0,|\nu| \geqq N>0$ and $x$ is real, then

$$
\left|D_{\nu}(x \eta)\right| \leqq A_{N}|\exp (M+E(\nu, x))| \text {. }
$$

For the similar consideration of $D_{\nu}(x \bar{\eta})$, the parts played by $\theta= \pm \frac{1}{4} \pi$ are interchanged. Hence (25), (26) are valid when $\eta$ is replaced by $\bar{\eta}$, while a result like $(27)$ is valid, when $\operatorname{Im} \nu \geqq 0$; and replacing $\nu$ by $-\nu-1$, we have: If $\operatorname{Rl} \nu \geqq-1, \operatorname{Im} \nu \leqq 0,|\nu+1| \geqq N \geqq 0$ and $x$ is real, then

$$
\begin{aligned}
\left|D_{-\nu-1}(x \eta)\right| \leqq A_{N} \mid \exp \left\{-\frac{1}{2}(\nu+1)\right. & \log (\nu+1) \\
& +\nu+1+E(-\nu-1, x)\} \mid
\end{aligned}
$$


Returning to the case $z=x \eta$, we now show that (26) is valid when $x<0$, provided Rlv $\geqq-1$. We have

$\left|2 e^{a} \operatorname{sech} a\right|^{\frac{1}{2}}=\left|e^{a}\right|\left|2 e^{-a} \operatorname{sech} a\right|^{\mid}=\left|e^{a}\right|\left|1+e^{2 a}\right|^{-1}$,

and hence, from (3), (23),

$$
\begin{aligned}
\left|D_{v}(x \eta)\right| & \leqq A_{\nu}\left|\exp \left\{M+\beta(1-\mu \cos 2 \theta)+\mu\left(\gamma \sin 2 \theta-\frac{1}{2} \sin 2 \gamma\right)\right\}\right| \\
& \times \begin{cases}\left|e^{-a} \nu^{b}\right|, & \text { for }\left|1+e^{-2 a}\right| \leqq|\nu|^{-\frac{1}{3}}, \\
\left|1+e^{2 a}\right|^{-\frac{1}{2}}, & \text { for }\left|1+e^{-2 a}\right|>|\nu|-\frac{1}{y} .\end{cases}
\end{aligned}
$$

Suppose for definiteness that $|v| \geqq \frac{1}{2}$. Then if $\left|1+e^{-2 a}\right| \leqq|v|-\frac{1}{s}$ we have $\left|e^{-2 a} \nu^{\frac{1}{3}}\right| \leqq\left(|\nu|^{-1}+1\right)|\nu|^{\frac{1}{3}}=\left(1+|\nu|^{\frac{1}{b}}\right) \leqq\left(1+2^{\frac{1}{3}}\right)|\nu|^{\frac{1}{b}}$

But if $\quad\left|1+e^{2 a}\right|<2-1|v|^{-\frac{1}{3}}$,

$$
\leqq 2^{3}|v|^{\frac{3}{5}}
$$

then $\quad\left|e^{2 \alpha}\right|>1-2-\frac{1}{\mid}|\nu|-1>\frac{1}{2}$,

and so $\left|1+e^{-2 a}\right|<2.2^{-\frac{1}{3}}|v|^{-\frac{1}{8}}<|\nu|^{-\frac{1}{3}}$.

Hence if $\left|1+e^{-2 a}\right|>|\nu|^{-\frac{1}{3}}$ we have $\left|1+e^{2 a}\right|-1<2^{2}|\nu|^{\frac{1}{3}} ;$ and for the second factor in (29) we can always take $2^{\frac{3}{3}}|\nu|^{h}$, provided $|\nu| \geqq \frac{1}{2}$.

Regarding the first factor in (29), we have $\beta \leqq 0$ for $x \leqq 0$, while $\mu \cos 2 \theta$ is the real part of $-\nu$. Hence, if $\mathrm{Rl} \nu \geqq-1$, $\beta(1-\mu \cos 2 \theta) \leqq 0 . \quad$ Further,

$$
d\left(\gamma \sin 2 \theta-\frac{1}{2} \sin 2 \gamma\right) / d \gamma=\sin 2 \theta-\cos 2 \gamma,
$$

which is negative for the relevant range of $\gamma$, viz. $0 \geqq \gamma>-\left(\frac{1}{4} \pi-\theta\right)$. Hence as $x$ decreases from $0, \mu(\gamma \sin 2 \theta \ldots$ and it is never greater than

$$
\mu\left\{\frac{1}{2} \sin \left(\frac{1}{2} \pi-2 \theta\right) \cdots\left(\frac{1}{4} \pi-\theta\right) \sin 2 \theta\right\},
$$

$=\frac{1}{2} \mu \cos 2 \theta\left\{1-\left(\frac{1}{2} \pi-2 \theta\right) \cot \left(\frac{1}{2} \pi-2 \theta\right)\right\} \leqq \frac{1}{2} \mathrm{RI}(-\nu) \leqq \frac{1}{2}$.

Hence (29) gives : if $-1 \leqq \mathrm{Rl} \nu \leqq 0,|\nu| \geqq \frac{1}{\text { and }} x \leqq 0$, then

$$
\left|D_{\nu}(x \eta)\right| \leqq A\left|\nu^{\frac{2}{2}} \exp M\right|
$$

there is of course a similar result for $v \mid \geqq N>0$ with the absolute constant ${ }^{1} A$ replaced by $4 \ldots$.

Finally, we note that, when $v^{\frac{1}{1}} / z^{3} \mid$ is large, (2) gives

$$
a=? z(-\nu)^{-\frac{1}{2}}+O\left(z^{3} v^{-3}\right) \text {, }
$$

so that $\nu(\sinh a \cosh a+a)=-z(-\nu)^{\frac{1}{2}}+O\left(z^{3} v^{-\frac{1}{2}}\right)$.

Hence, for $\left|\nu^{\frac{1}{2}} z^{-3}\right| \geqq 2$ and $|\nu| \geqq \frac{1}{2}$, say,

$$
\exp E(\nu, z) \leqq A\left|\exp \left\{-z(-\nu)^{\frac{1}{2}}\right\}\right|
$$

where $A$ is an absolute constant; and since $a$ is small, (3) gives

$$
\left.\left|D_{\nu}(z)\right| \leqq A\right\} \exp \left\{M-z(-\nu)^{\frac{1}{3}}\right\} \mid
$$

1 Here, and below, the usual convention is made that an $A$ or $A_{N}$ is not necessarily the same at each occurrence. 
4. Let us now assume that $f(x)$ is of bounded variation in any finite interval and that $\int_{-\infty}^{\infty}|f(x)| d x$ exists. Then from (26), for $\mathrm{Rl} \nu \leqq 0$ and $|\nu| \geqq N>0$, with $b^{\prime}>b \geqq 0$,

$$
\left|\int_{b}^{b^{\prime}} f(t) D_{\nu}(t \eta) d l\right| \leqq A_{N}\left|\nu^{\frac{1}{c}} \exp \left(\frac{1}{2} \nu \log (-\nu)-\frac{1}{2} \nu\right)\right| \int_{b}^{b^{\prime}}|f(t)| d t,
$$

and so $\int_{0}^{\infty} f(t) D_{\nu}(t \eta) d t$ converges uniformly in any compact part of the region $\mathrm{Rl} \nu \leqq 0,|\nu| \geqq N>0$. Hence this integral is an analytic (regular) function of $\nu$ in $\mathrm{Rl} \nu<0$, and is continuous up to $\mathrm{Rl}_{\nu}=0$, except perhaps at $\nu=0$. Similarly $\int_{-\infty}^{0} f(t) D_{\nu}(-t \eta) d t$ is regular in $\mathrm{Rl} \nu<0$; and, replacing $\nu$ by $-\nu-1$ and $\eta$ by $\bar{\eta}$, we see that

are regular in $\mathrm{RI} \nu>-1$.

$$
\int_{0}^{\infty} f(t) D_{-\eta-1}(\bar{\eta}) d t, \quad \int_{-\infty}^{0} f(t) D_{-\nu-1}(-\bar{t}) d t
$$

4.1 Now let $x$ be arbitrary but fixed, and let $b>x, b>0$. We shall prove that

$$
\int_{-\frac{1}{2}-i \infty}^{-\frac{1}{t}} d v \int_{b}^{\infty} \Gamma(-v) \eta f(t) D_{v}(-x \eta) D_{v}(t \eta) d t
$$

exists, for integration along $\mathrm{Rl} \nu=-\frac{1}{2}$. For this purpose we use the fact, just proved, that the $\nu$-integrand is regular in $\mathrm{Rl} \nu<0$; this enables us to estimate $\int_{-\frac{1}{2}-i u^{2}}^{-\frac{1}{2}-i s}(\ldots) d \nu$ by deforming the straight path into the left half-plane.

Writing $\nu=r^{2} \exp i\left(-\frac{1}{2} \pi-2 \phi\right)$, we choose a constant $\phi_{0}$, say $\phi_{0}=\frac{1}{4} \pi$, and take for the deformed path
(i) the arc $r=u_{i}=\left(\frac{1}{4}+u^{4}\right)^{\ddagger}, 0<\phi \leqq \phi_{0}$,
(ii) the radius $\dot{\phi}=\phi_{0}, u_{1} \geqq r \geqq s_{1}=\left(\frac{1}{4}+s^{4}\right)^{4}$,
(iii) the arc $r=s_{1}, \phi_{0} \geqq \phi>0$.

The distinction between $u, u_{1}$ and between $s, s_{1}$ are trivial, and will for simplicity be neglected below.

Let $y$ be any real number exceeding $b$. Then from (10), for $\mathrm{Rl} \nu \leqq 0$ and $|\nu| \geqq N_{y}$,

$$
\left|\int_{b}^{y} \Gamma(-\nu) f(t) D_{v}(-x \eta) D_{v}(t \eta) d t\right| \leqq\left. C_{y}\right|^{\nu^{-1}} \exp \left\{-(-\nu)^{\mathbf{t}_{\eta}} \eta(b-x)\right\} \mid,
$$

where $N_{y}, C_{y}$ depend upon ${ }^{1} y$; and here $(-v)^{\frac{1}{\eta}} \eta=r \exp i\left(\frac{1}{4} \pi-\phi+\frac{1}{4} \pi\right)$,

1 In fact, as in the proof of (31), the choice $N_{y}=\max \left(4 y^{6}, 4 x^{6}\right)$ is suitable, but no such explicit estimate is needed in the argument. 
so that

$$
\left|\int_{b}^{y} \Gamma(-v) f(t) D_{v}(-x \eta) D_{\nu}(\eta \eta) d t\right|<C_{y} r^{-2} \exp \{-(2 / \pi) r(b-x) \phi\} .
$$

Also, using (27) for $D_{v}(t \eta)$ and (32) for $D_{\nu}(-x \eta)$ we have for $\operatorname{Rl} \nu \leqq 0$, $\operatorname{Im} v \leqq 0$ and $\left|v^{\frac{1}{2}} x-3\right| \geqq 2$,

$$
\begin{aligned}
& \left|\int_{y}^{\infty} \Gamma(-\nu) \eta f(t) D_{\nu}(-x \eta) D_{\nu}(t \eta) d t\right| \\
& \leqq A\left|\Gamma(-\nu) e^{2 M I}\right|\left|\exp \left\{x \eta(-\nu)^{\frac{t}{2}}+E(\nu, y)\right\}\right| \int_{y}^{\infty}|f(t)| d t \\
& \quad<A\left|\nu^{-\frac{1}{2}} \exp \left\{x \eta(-\nu)^{\frac{1}{2}}+E(\nu, b)\right\}\right| \int_{\nu}^{\infty}|f(t)| d t
\end{aligned}
$$

since, by $(24), E(\nu, y)<E(\nu, b)$ and

$$
\left|\Gamma(-v) e^{2 M}\right|=|\Gamma(-\nu) \exp \{\nu \log (-\nu)-v\}| \leqq A|\nu|^{-\frac{1}{2}} .
$$

Again, estimating $E(\nu, b)$ from (31), we have, for $\mathrm{Rl} \nu \leqq 0$ and $\left|\nu^{\frac{1}{2}} b^{-3}\right| \geqq 2$, $\left|\exp \left\{x \eta(-\nu)^{\frac{1}{2}}+E(\nu, b)\right\}\right| \leqq A\left|\exp \left\{-(b-x) \eta(-\nu)^{\frac{1}{2}}\right\}\right|$

so that

$$
<A \exp \{-(2 / \pi) r(b-x) \phi\} \text {, }
$$

$$
\begin{aligned}
\mid \int_{y}^{\infty} \Gamma(-\nu) \eta f(t) D_{\nu}( & -x \eta) D_{\nu}(t \eta) d t \mid \\
& <A r^{-1} \exp \{-(2 / \pi) r(b-x) \phi\} \int_{y}^{\infty}|f(t)| d t
\end{aligned}
$$

This is established, with $A$ an absolute constant, provided Rl $v \leqq 0$, $\operatorname{Im} \nu \leqq 0,\left|\nu^{\frac{1}{2}}\right| \geqq 2 \max \left(b^{3},|x|^{3}\right)$, and $y \geqq b$, and it is vital that the lower bound for $\left|\nu^{\frac{1}{2}}\right|$ serves for arbitrarily large $y$.

By adding (34), (35) we have an estimate for the integrand, $G(\nu)$ say, of (33) on the deformed path. Since $\nu=-r^{2} i e^{-2 i \phi}$, the contribution to the modulus of the integral from each arc $r=$ constant is hence less than

$\int_{0}^{\phi_{0}} \exp \left\{-\frac{2}{\pi} r(b-x) \phi\right\} \cdot\left\{\frac{C_{y}}{r^{2}}+\frac{A Y}{r}\right\} 2 r^{2} d \phi,<\frac{\pi}{b-x}\left(\frac{C_{y}}{r}+A Y\right)$, and the contribution from the radius $\phi=\phi_{0}$ is less than

$$
\int_{s}^{u} \exp \left\{-\frac{2}{\pi} r(b-x) \phi_{0}\right\} \cdot\left\{\frac{C_{y}}{r^{2}}+\frac{A Y}{r}\right\} 2 r d r,<\frac{\pi}{(b-x) \phi_{0}}\left(\frac{C_{y}}{s}+A Y\right),
$$

where we have taken $u>s$ and written $Y=\int_{y}^{\infty}|f(t)| d t$. Hence, for $s^{2} \geqq N_{y}, s^{2} \geqq 4 b^{6}$, and $u^{2}>s^{2}$,

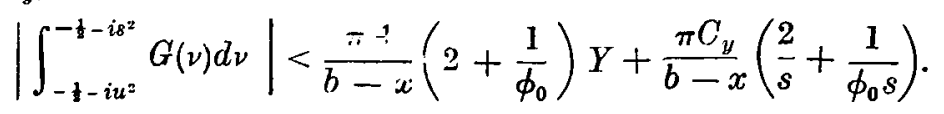

The right-hand side is made less than any positive $\epsilon$ by first choosing a $y$ for which 


$$
\frac{\pi A}{b-x}\left(2+\frac{1}{\phi_{0}}\right) Y<\frac{1}{2} \epsilon
$$

and then choosing $s$ such that

$$
\frac{\pi C_{y}}{b-x}\left(\frac{2}{s}+\frac{1}{\phi_{0} s}\right)<\frac{1}{2} \epsilon, \quad s^{2}>N_{y}, s^{2}>4 b^{6} .
$$

This being true for any $u$ exceeding $s$, the existence of the integral (33) is established.

It follows that, as $y \rightarrow \infty$,

$$
\begin{aligned}
& \int_{-\frac{1}{2}-i}^{-\frac{1}{2}} d \nu \int_{b}^{y} \Gamma(-\nu) \eta f(t) D_{\nu}(-x \eta) D_{\nu}(t \eta) d t \\
& \rightarrow \int_{-1-i \infty}^{-\frac{1}{2}} d \nu \int_{b}^{\infty} \Gamma(-\nu) \eta f(t) D_{\nu}(-x \eta) D^{\nu}(t \eta) d t,
\end{aligned}
$$

i.e. that

$$
\left\{\int_{-\frac{1}{2}-i \infty}^{-\frac{1}{2}-i \delta^{2}}+\int_{-\frac{1}{2}-i \delta^{\prime \prime}}^{-\frac{1}{2}}\right\} d \nu \int_{y}^{\infty} \Gamma(-v) \eta f(t) D_{v}(-x \eta) D_{\nu}(t \eta) d t \rightarrow 0 .
$$

For the first $\imath$-integral, we may estimate the integrand by (35) provided $s=2 \max \left(b^{3},\left.x\right|^{3}\right)$, and the preceding work gives

$$
\left|\int_{-1-i \infty}^{-\frac{1}{2}-i s^{2}}(\ldots) d \nu\right|<\frac{\pi A}{b-x}\left(2+\frac{1}{\phi_{0}}\right) \int_{y}^{\infty}|f(t)| d t
$$

while for the sernnd integral, the crude estimate (25) gives

$$
\begin{aligned}
& \left|\int_{-\frac{1}{2}-i \varepsilon^{2}}^{-\frac{1}{2}}(\ldots) d \nu\right| \\
& <\left(A_{\frac{1}{2}}\right)^{2} \int_{y}^{\infty}|f(t)| d t\left|\int_{-\frac{1}{2}-i s^{2}}^{-\frac{1}{2}} \Gamma(-\nu) \exp \{2 M+E(\nu,-x)+E(\nu, b)\} d \nu\right| \\
& =C(s) \int_{y}^{\infty}|f(t)| d t ;
\end{aligned}
$$

and since $s$ is fixed independently of $\nu,(37)$ is evident.

In a similar way we prove that, as $y \rightarrow \infty$,

$$
\begin{aligned}
\int_{-\frac{1}{2}-i_{\infty}}^{-\frac{1}{2}} d \nu \int_{b}^{\nu} \Gamma(\nu & +1) \bar{\eta} D_{-\nu-1}(-x \bar{\eta}) D_{-\nu-1}(\bar{\eta}) d t \\
& \rightarrow \int_{-\frac{1}{2}-i_{\infty}}^{-\frac{1}{2}} d \nu \int_{b}^{\infty} \Gamma(\nu+1)_{\bar{\eta}} D_{-\nu-1}(-a \bar{\eta}) D_{-\nu-1}(t \bar{\eta}) d t
\end{aligned}
$$

The essential facts are that the integrand is regular in $\mathrm{Rl}_{\nu}>-1$ and that for $\operatorname{Im} v \leqq 0$ we have for the inner integral an estimate based on (28); the analogue of (36) is proved by deforming the path into the half-plane $\mathrm{RI}_{\nu}>-1$.

\subsection{Next we prove that}

$$
\begin{aligned}
\int_{-\frac{1}{2}}^{-\frac{1}{2}+i \infty} d \nu \int_{b}^{\infty} f(t)\left\{\Gamma(-\nu) \eta D_{\nu}(-x \eta) D_{\nu}(t \eta)\right. \\
+\Gamma(\nu+1) \vec{\eta} D_{-\nu-1}\left(-(x \bar{\eta}) D_{-\nu-1}(\overline{t \eta})\right\} d t
\end{aligned}
$$


exists, and is equal to the limit as $y \rightarrow \infty$ of the corresponding expression with the inner integral from $b$ to $y$. If, by way of exploration, we estimate the integrand by means of (7), we find for the two terms in $\{\ldots\}$ the respective principal parts (for $v \sim i \infty$ )

$$
i_{i} \pi / 2{ }^{\prime} \exp \left\{\nu^{\prime}(x-t)\right\}, \quad-i_{i} \pi / 2 \nu^{\prime \frac{1}{2}} \exp \left\{\nu^{\prime \frac{1}{t}}(x-t)\right\} ;
$$

so that they cancel (to this order), and in fact the terms separately give divergent integrals. It is essential therefore to transform the integrand as in the passage from (15) to (16); it is sufficient to prove that

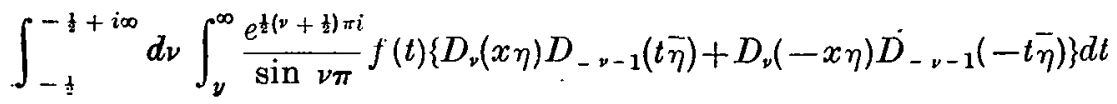

exists and tends to 0 as $y \rightarrow \infty$.

Irrespective of the signs of $x, y$, we can estimate $D_{v}( \pm x \eta)$ by (26) or (30), and $D_{-v-1}( \pm t \bar{\eta})$ by the analogous formula; hence the integrand of $(40)$ is less in absolute value than

$A \mid \operatorname{cosec} \nu \pi .\left(\nu^{2}+\nu\right) \frac{1}{\pi} \exp \left\{\frac{1}{2} \nu \pi i+\frac{1}{2} \nu \log (-\nu)-\frac{1}{2} \nu-\frac{1}{2}(\nu+1) \log (\nu+1)\right.$

Since $\operatorname{Im} v>0$ on the path, we write

$$
\left.+\frac{1}{2}(\nu+1)\right\}\left|\int_{y}^{\infty}\right| f(t) \mid d t
$$

$$
\log (\nu+1)=\log \nu+\log \left(1+\nu^{-1}\right)=\log (-\nu)+\pi i+\nu^{-1}+O\left(\nu^{-2}\right), .
$$

and the argument of the exponential reduces to $-\frac{1}{2} \log (-\nu)+O\left(\nu^{-1}\right)$. Hence for $|v| \geqq 2$, say, the integrand is less in absolute value than

$$
A\left|\nu^{-\frac{1}{6}} e^{\nu \pi i}\right| \int_{y}^{\infty}|f(t)| d t
$$

while the same form is valid on the parts of the path for which $|\nu|<2$, since there the functions of $v$ that are concerned lie between positive bounds. It follows that the integral (40) is absolutely convergent, and tends to 0 as $y \rightarrow \infty$.

Combining this result with (37), (38) we see that, for $b>x, b>0$, $\int_{-\frac{1}{2}-i \infty}^{-\frac{1}{2}+i \infty} d \nu \int_{b}^{\infty} f(t)\left\{\Gamma(-\nu) \eta D_{\nu}(-x \eta) D_{v}(t \eta)\right.$

$$
\left.+\Gamma(\nu+1) D_{-\nu-1}\left(-\overline{x_{\eta}}\right) D_{-\nu-1}(\ell \eta)\right\} d t
$$

exists, and is equal to the limit as $y \rightarrow \infty$ of the corresponding expression with the inner integral from $b$ to $y$. But for any finite $y$ the latter expression is equal to zero, by Theorem 1, since this is true for any finite $b$ exceeding $x$. Hence the expression (41) is equal to zero, provided $b>x, b>0$. 
4.3 To prove the existence and vanishing of the expression corresponding to (41) with the inner integral from $-\infty$ to $a, a<x$, the treatment of $\int_{-\frac{1}{2}}^{-\frac{1}{2}+i \infty}(\ldots) d \nu$ is the same as in $\S 4.2$; but for $\int_{-\frac{1}{2}-i \infty}^{-\frac{1}{2}}(\ldots) d \nu$ the form $(41)$ is unsuitable because each term of the integrand is regular only in the strip $-1<\mathrm{Rl} \nu<0$. We can however transform the integrand to the form shown in (17), and for this thetreatment of $\S 4.1$ is applicable.

Finally we add to the right-hand side of (15) the vanishing integral (41) and its analogue, and have:

THEOREM 2. If $f(x)$ is of bounded variation in any finite interval and absolulely integrable in $(-\infty, \infty)$, then for any real $x$

$$
\begin{aligned}
& -4 \pi i f(x)=\int_{-\frac{1}{2}-i \infty}^{-\frac{1}{2}+i \infty} \frac{e^{\left.\frac{1}{k^{(v+1}}\right) \pi i} d v}{\sin \nu \pi} \int_{-\infty}^{\infty} f(t)\left\{D_{1}(x \eta) D_{-\nu-1}(t \bar{\eta})\right. \\
& \left.+D_{\nu}(-x \eta) D_{-\nu-1}\left(-t_{\bar{\eta}}\right)\right\} d t
\end{aligned}
$$

where $\eta=e^{\pi i / 4}, \bar{\eta}=e^{-\pi i / 4}$.

5. Lxamples. The formula (42) is valid for the function

$$
f(x, y)=\exp \left\{-\frac{1}{4} i\left(x^{2}-y^{2}\right) \cos a-\frac{1}{2} i x y \sin a\right\}
$$

which specifies a plane wave in parabolic coordinates $x, y$, provided $a$ is not a multiple of $\pi$. If we take either $x$ or $y$ as the variable, the $t$-integrals required for (42) exist; but since $f$ is not absolutely integrable we cannot appeal to Theorem 2, and the formula which is. obtained, viz.

$$
\begin{aligned}
-2(2 \pi)^{\frac{1}{2}} i f(x, y)=\int_{-\frac{1}{2}-i \infty}^{-\frac{1}{2}+i \infty} \frac{d \nu}{\sin \nu \pi} & \frac{\left(\left(\tan \frac{1}{3} \alpha\right)^{\nu}\right.}{\left(\cos \frac{1}{2} \alpha\right.} D_{\nu}(-x \eta) D_{-\nu-1}(y \eta) \\
& \left.+\frac{\left(\cot \frac{1}{2} \alpha\right)^{\nu}}{\sin \frac{1}{2} \alpha} D_{-\nu-1}(x \eta) D_{\nu}(-y \eta)\right)
\end{aligned}
$$

requires independent verification. It has been proved by Erdélyi ${ }^{1}$ in a slightly different notation; the form (44) is valid for $0<13 l a<\pi$ and any complex $x, y$.

The two terms on the right of (44) both give convergent integrals. and it is of interest that these represent Sommerfeld's two diffracted. plane waves. $^{2}$ To prove this, let $a$ be real, $0<a<\pi$, and let

1 Proc. Roy. Soc. Edinburgh, 61 (1941), 61-70.

2 See, for example, Baker and Copson, The Mathematical Theory of Huygens Principle, Chap. IV ; the function defined by (1.65) on p. 140 agrees, to a constant. factor, with $J$ as given by my equation (47). 


$$
J=\int_{-i-i \infty}^{-\frac{1}{2}+i \infty} \frac{d \nu}{\sin \nu \pi} \frac{\left(\tan \frac{1}{2} \alpha\right)^{v}}{\cos \frac{1}{2} \alpha} D_{s}\left(-x_{\eta}\right) D_{-\nu-1}(y \eta) ;
$$

and let the integral shown in the footnote on page 51 be substituted for each $D$-function, so that

$$
J=\frac{i}{2 \pi} \int_{-\frac{1}{2}-i \infty}^{-\frac{1}{2}+i \infty} \frac{d \nu}{\sin \nu \pi} \int_{i_{c}-\infty}^{i c+\infty} d s \int_{i c-\infty}^{i c+\infty} \frac{\left(\tan \frac{1}{2} \alpha\right)^{\nu} s^{\nu}}{\cos \alpha t^{\nu+1}} \exp \psi d t,
$$

where

$$
\psi=\frac{1}{4} i\left(x^{2}+y^{2}\right)+i \eta(y t-x s)-\frac{1}{2}\left(s^{2}+t^{2}\right) .
$$

Since all the integrals converge exponentially we can take the $\nu$-integral first, and use the result

$$
\int_{-i-i \infty}^{-\frac{1}{2}+i \infty} \frac{\exp (\nu \log p)}{\sin \nu \pi} d v=-\frac{2 i}{1+p},
$$

valid for $|\operatorname{amp} p|<\pi$; this condition being satisfied for all the values of $p$ in question, viz. $s \tan \frac{1}{2} a / t, t \cot \frac{1}{2} a / s$, provided $s, t$ lie on straight paths $\operatorname{Im} s=c, \operatorname{Im} t=c$ in the upper half-plane. Hence we get

$$
\pi J=\int_{i c-\infty}^{i c+\infty} d s \int_{i c-\infty}^{i c+\infty} \frac{\exp \psi d t}{s \sin \frac{1}{2} \alpha+t \cos \frac{1}{2} \alpha} .
$$

It is easy to justify the change of variables

$$
u=s \cos \frac{1}{2} \alpha-t \sin \frac{1}{2} \alpha, \quad v=s \sin \frac{1}{2} \alpha+t \cos \frac{1}{2} \alpha,
$$

which gives $\pi J=\int_{i l-\infty}^{i l+\infty} \exp \psi_{1} d u \int_{i k-\infty}^{i k+\infty} \exp \psi_{2} \frac{d v}{v}$,

where

$$
\begin{aligned}
& k=c\left(\sin \frac{1}{2} \alpha+\cos \frac{1}{2} \alpha\right)>0, \\
& l=c\left(\cos \frac{1}{2} \alpha-\sin \frac{1}{2} x\right),
\end{aligned}
$$

and $\psi_{1}=-\frac{1}{2}\left\{u+i \eta\left(x \cos \frac{1}{2} a+y \sin \frac{1}{2} \alpha\right)\right\}^{2}-\frac{1}{4} i\left(x \cos \frac{1}{2} a+y \sin \frac{1}{2} a\right)^{2}$,

$\psi_{2}=-\frac{1}{2} v^{2}-i v \eta\left(x \sin \frac{1}{2} \alpha-y \cos \frac{1}{2} \alpha\right)+\frac{1}{4} i\left(x \sin \frac{1}{2} \alpha-y \cos \frac{1}{2} \alpha\right)^{2}$.

On the right of (46) we thus have the product of two simple integrals; in particular the $v$-integral is equal to $-i(2 \pi)^{\frac{1}{t}} D_{-1}\left\{\eta\left(y \cos \frac{1}{2} a-x \sin \frac{1}{2} a\right)\right\}$. Hence we find

$J=-2 i D_{0}\left\{\eta\left(x \cos \frac{1}{2} a+y \sin \frac{1}{2} \alpha\right)\right\} D_{-1}\left\{\eta\left(y \cos \frac{1}{2} a-x \sin \frac{1}{2} \alpha\right)\right\} ;$

and this is the representation, in parabolic coordinates, of Sommerfeld's. diffracted plane wave.

An example of a discontinuous function, represented for real values only of the argument, is provided by

$$
\left.\begin{array}{ll}
f(x)=D_{\mu}(x \eta) & (x>0) \\
f(x)=0 & (x<0)
\end{array}\right\} .
$$

Since $D_{\mu}(x \eta) \sim e^{-t i x^{2}}(x \eta)^{\mu}$ for $x \sim \infty$, the hypotheses of Theorem 2 
are satisfied if $\mathrm{Rl}_{\mu}<-1$. The result can be written in the two forms

$$
\begin{aligned}
& 2 \frac{3}{2} \pi i f(x)=\int_{-\frac{1}{2}-i \infty}^{-\frac{1}{2}+i \infty} \frac{d \nu}{\nu-\mu}\left\{2^{\frac{1}{(2(\mu-\nu-1)} D_{\nu}(-x \eta)}\left(\frac{\Gamma\left(-\frac{1}{2} \nu\right)}{\Gamma\left(-\frac{1}{2} \mu\right)}-\frac{\Gamma\left(\frac{1}{2}-\frac{1}{2} \nu\right)}{\Gamma\left(\frac{1}{2}-\frac{1}{2} \mu\right)}\right)\right. \\
& \left.+2^{\frac{1}{3(\mu+v)} D_{-\nu-1}(-x \bar{\eta})}\left(\frac{\Gamma\left(1+\frac{1}{2} \nu\right)}{\Gamma\left(\frac{1}{2}-\frac{1}{2} \mu\right)}-i \frac{\Gamma\left(\frac{1}{2}+\frac{1}{2} \nu\right)}{\Gamma\left(-\frac{1}{2} \mu\right)}\right)\right\} \\
& 2^{\frac{i}{2}} \pi i f(x)=\int_{-\frac{1}{2}-i \infty}^{-\frac{1}{2}+i \infty} \frac{d \nu}{\nu-\mu}\left\{2^{\frac{1}{2}(\mu-\nu-1)} D_{\nu}(x \eta)\left(\frac{\Gamma\left(-\frac{1}{2} \nu\right)}{\Gamma\left(-\frac{1}{2} \mu\right)}+\frac{\Gamma\left(\frac{1}{2}-\frac{1}{2} \nu\right)}{\Gamma\left(\frac{1}{2}-\frac{1}{2} \mu\right)}\right)\right. \\
& \left.-2^{\frac{1}{(1}(\mu+\nu)} D_{-\nu-1}(\overline{x \eta})\left(\frac{\Gamma\left(1+\frac{1}{2} \nu\right)}{\Gamma\left(\frac{1}{2}-\frac{1}{2} \mu\right)}+i \frac{\Gamma\left(\frac{1}{2}+\frac{1}{2} \nu\right)}{\Gamma\left(-\frac{1}{2} \mu\right)}\right)\right\} \text {. }
\end{aligned}
$$

These forms answer respectively to the general forms (15), (17), and the two integrands are identical.

The truth of this formula is easily checked, and it is indeed valid under the sole restriction that $\mu$ be not zero or a positive integer, provided the path of integration is so deformed that the point $\nu=\mu$ lies on its left while the points $\nu=0,1,2, \ldots$ remain on its right. By use of (1) it is easily shown that, for the first term on the right of (49), the path can be closed at infinity in the left half-plane, provided $\operatorname{amp} x$ is between $\frac{1}{2} \pi, \pi$ inclusive; and for the second term the path can be closed in the right half-plane, provided amp $x$ is between $-\frac{1}{2} \pi$, - $\pi$ inclusive. Hence for $x$ real and negative the right-hand side of (49) is zero, the pole of $(\nu-\mu)^{-1}$ in the first term being cancelled by the second factor. For $x$ real and positive the form (50) is similarly used, and the residue of the first term at $\nu=\mu$ gives $f(x)=D_{\mu}(x \eta)$, in agreement with (48).

If $\mu$ is a non-negative integer, the terms in $\Gamma\left(-\frac{1}{2} \nu\right)$ or $\Gamma\left(\frac{1}{2}-\frac{1}{2} \nu\right)$ are absent from (49) and (50), according as $\mu$ is even or odd. In both cases we find, in place of $(48)$,

$$
\left.\begin{array}{ll}
f(x)=-\frac{1}{2} D_{\mu}(x \eta) & (x<0) \\
f(x)=\frac{1}{2} D_{\mu}(x \eta) & (x>0)
\end{array}\right\} .
$$

In the general case, if we take the point $\nu=\mu$ to the right of the path, the function represented by (49) or (50) is again discontinuous; in place of (48) we have

$$
\begin{array}{ll}
f(x)=\frac{1}{2} \exp \left(-\frac{1}{2} i \mu \pi\right) \Gamma(\mu+1) D_{-\mu-1}(x \bar{\eta}) & (x>0)) \\
f(x)=-\frac{1}{2} \exp \left(\frac{1}{2} i \mu \pi\right) \Gamma(\mu+1) D_{-\mu-1}(-x \bar{\eta}) & (x<0) !
\end{array} .
$$

Finally, if in (49), (50) we replace $x$ by $-x$, we get the representation of the function $f_{1}(x)$ for which

$$
\left.\begin{array}{ll}
\dot{f}_{\mathbf{1}}(x)=D_{\mu}(-x \eta) & (x<0) \\
f_{1}(x)=0 & (x>0)
\end{array}\right\} .
$$


It is easy now to prove that the formula (A) is valid under the condition (D) of $\S 1$. For (i), we have just verified (A) for the functions given by (48), (5l), provided Rl $\mu<-\frac{1}{2}$ (this condition being imposed so that we can use the original path of integration $\left.\mathrm{Rl}_{\nu}=-\frac{1}{2}\right)$; and (ii), for $x \sim+\infty$,

$$
D_{\mu}(x \eta) \sim e^{-i x 2 / 4}\left\{(x \eta)^{\mu}-\frac{1}{2} \mu(\mu-1)(x \eta)^{\mu-2}\right\} .
$$

Hence a function satisfying (D) can be reduced to an absolutely integrable function, for which Theorem 2 is valid, by adding suitable multiples of the functions (48), (51).

The University,

Melbourne, Australta. 